\title{
Das MMM2 - Ein regionalgeschichtliches Onlinelexikon der Arbeitsgemeinschaft für mittelrheinische Musikgeschichte
}

Axel Beer, Martin Bierwisch, Kristina Krämer, Mainz

DOI: $10.25366 / 2020.108$

Zitation: Axel Beer, Martin Bierwisch, Kristina Krämer, „Das MMM2 - Ein regionalgeschichtliches Onlinelexikon der Arbeitsgemeinschaft für mittelrheinische Musikgeschichte", in: Brückenschläge zwischen Musikwissenschaft und Informatik. Theoretische und praktische Aspekte der Kooperation, in Verbindung mit der Fachgruppe Digitale Musikwissenschaft hrsg. von Stefanie Acquavella-Rauch, Andreas Münzmay und Joachim Veit (= Musikwissenschaft: Aktuelle Perspektiven. Bericht über die Jahrestagung der Gesellschaft für Musikforschung 2019 in Paderborn und Detmold, Bd. 3), Detmold, Musikwissenschaftliches Seminar der Universität Paderborn und der Hochschule für Musik Detmold, 2020, S. 199-205, DOI: 10.25366/2020.108 


\title{
Das MMM2 - Ein regionalgeschichtliches Onlinelexikon der Arbeitsgemeinschaft für mittelrheinische Musikgeschichte
}

\author{
AXEL BEER, MARTIN BIERWISCH, KRISTINA KRÄMER, MAINZ
}

Das Lexikon wurde in einer ersten Auflage von Hubert Unverricht (1927-2017) in den Jahren 1974 und 1981 als Band 21 und 22 der Beiträge zur mittelrheinischen Musikgeschichte mit 83 Artikeln herausgegeben. Inzwischen (dies nach Vorüberlegungen, die bis 2001 zurückreichen und auf eine gedruckte Fortsetzung abzielten) ermöglichten insbesondere die technischen Voraussetzungen, dass der Plan einer erweiterten Neuauflage Realität werden konnte:

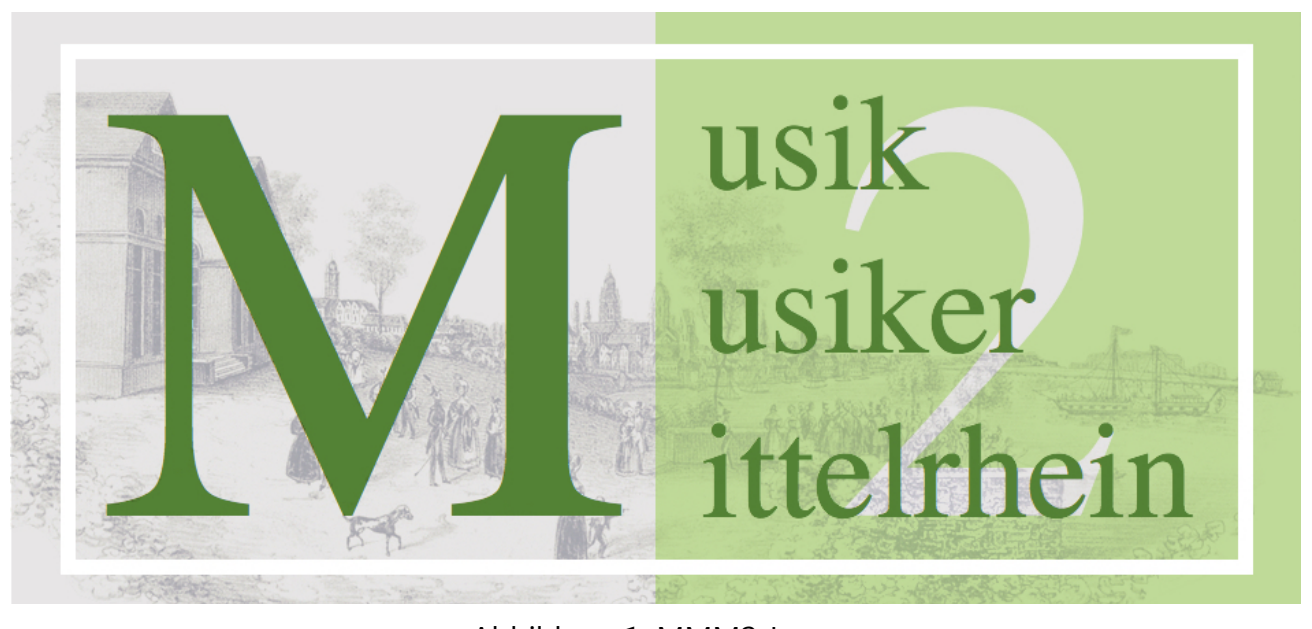

Abbildung 1: MMM2-Logo

Nachdem 2017 die Entscheidung zur Onlinepublikation getroffen worden war, wurden im Laufe der folgenden Monate die Website erstellt, außerdem ältere Artikel revidiert und neue verfasst. Im Oktober 2018 ging das Lexikon mit 250 Artikeln online. Seitdem wuchs es stetig (Stand Sept. 2019: 450 Artikel; Mai 2020: 570 Artikel). Neben Prof. Dr. Axel Beer (JGU Mainz) als Herausgeber sind Kristina Krämer M.A., Martin Bierwisch M.A. und seit kurzem Simon Rech als Mitarbeiter mit redaktionellen und technischen Aufgaben betraut. Die personelle Nähe des Teams zur Universität Mainz ermöglicht eine Einbindung in die Lehre. So wurde im Wintersemester 2018/19 die Masterübung "Musik und Musiker am Mittelrhein. Ein Lexikon entsteht" angeboten, mit dem Ziel, den Studierenden praktische Erfahrungen in den Bereichen Lexikographie, Quellenkunde und Textgestaltung zu vermitteln. Darüber hinaus steuern zahlreiche Musik- und Lokalgeschichtsforscher*innen aus dem Umfeld der Arbeitsgemeinschaft für mittelrheinische Musikgeschichte Artikel bei. 


\section{Warum regionale Musikgeschichtsforschung?}

Noch immer hat man mit unterschiedlichen Formen des Naserümpfens zu rechnen, wenn es um regional ausgerichtete Musikforschung geht. Freilich: Landauf, landab begegnen wir unterschiedlichen Ausprägungen mehr oder weniger selbstgefälliger kultureller Nabelschau, deren Ergebnisse man ebenso belächeln wie (sei es aus Höflichkeit, sei es mit einer gewissen Anerkennung) als Beleg für ein kreatives gesellschaftliches Miteinander betrachten mag. Allerdings wäre es verfehlt, Gräben aufzuwerfen zwischen "seriöser" Wissenschaft, deren Gegenstand der räumlichen Zuordnung - vermeintlich - nicht bedarf, und einer Herangehensweise, die sich der Musikgeschichte einer Region verpflichtet fühlt. Jenes Gegeneinander ist zumal dann obsolet, wenn es eben nicht mittels diverser Rechtfertigungsstrategien darum geht, das regionale Geschehen und seine Protagonisten gleichsam in einem fiktiven Wettbewerb gegen die sogenannte Hochkultur antreten zu lassen. Ästhetische Maßstäbe sind es nicht, die über die Auswahl der Lemmata bestimmen; dass eine Person existiert, zudem komponiert, Instrumente gebaut oder Musik veröffentlicht hat, ist entscheidend. Und ebenso entscheidend wie auch die Grundlage unserer Konzeption bildend ist es, sich vorurteilsfrei, behutsam und durchaus respektvoll - das heißt weder mit verachtendem Naserümpfen, noch mit bildungsbürgerlicher Emphase - anzunähern. Dementsprechend folgt die Gestaltung der Artikel der Maßgabe, personengeschichtliche Fakten möglichst übersichtlich, mithin kurz und bündig, zu präsentieren und vor allem keinerlei künstlerische oder ästhetische Bewertung des jeweiligen Schaffens einfließen zu lassen. Also verstehen wir insbesondere die zweite Auflage des "MMM" als einen „Eitner für den Mittelrhein“, eine Dokumentation des Quellenrepertoires.

\section{Wen findet man im Lexikon?}

Komponisten*innen, Instrumentenbauer*innen, Musikpädagogen*innen, Wissenschaftler*innen oder auch Verleger*innen, die im mittelrheinischen Raum nicht nur tätig waren, sondern auch "Werke" in einem umfänglichen Sinne hinterlassen haben, sind vertreten. Hierbei ist neben der musikalischen "Prominenz" der Region ein vollkommen vergessener und in keinem anderen Lexikon zu findender Mainzer Musiklehrer, wie etwa Frohwald Thiemer, ${ }^{1}$ von dem eine Handvoll Kompositionen zu ermitteln war, ebenso selbstverständlich enthalten wie ein Niccolò Paganini, ${ }^{2}$ der in der Region konzertierte und einige Monate in Frankfurt weilte - dass Personen von überregionaler und allgemeiner Bekanntheit wie etwa Letzterer vor allem hinsichtlich ihrer Tätigkeit oder ihrer Präsenz im mittelrheinischen Raum Berücksichtigung finden, sei betont. Besonderes Gewicht wird gerade im Falle weniger oder bisher gar nicht geläufiger Namen neben

1 Axel Beer, Art. „Thiemer, Frohwald", in: Musik und Musiker am Mittelrhein 2 - Online, hrsg. im Auftrag der Arbeitsgemeinschaft für mittelrheinische Musikgeschichte von Axel Beer, Mainz 2018-2020, <http://mmm2.mugemir. de/doku.php?id=thiemer> (24.09.2019).

2 Ders., Art. „Paganini, Niccolò", in: MMM2, <http://mmm2.mugemir.de/doku.php?id=paganini> (29.03.2019). 
der Präsentation biographischer Fakten auf die Erfassung des kompositorischen Schaffens gelegt, wobei, sofern zu ermitteln, Fundorte unter Verwendung der RISM-Siglen angegeben sind. Lebende Personen werden nicht berücksichtigt.

Der zu betrachtende Raum umfasst zunächst das gesamte heutige Bundesland RheinlandPfalz, ferner das Saarland, von Hessen den Rheingau und Teile des ehemaligen Fürstentums Nassau, das frühere Großherzogtum Hessen-Darmstadt, von Baden-Württemberg die Teile der ehemaligen Kurpfalz, schließlich die im ehemaligen "Oberstift" des Kurfürstentums Mainz in Bayern (Unterfranken) gelegenen Gebiete. Städtische Zentren sind Mainz, Wiesbaden, Frankfurt a. M., Offenbach, Darmstadt, Aschaffenburg, Worms, Speyer, Koblenz und Trier.

\section{Quellen}

Für die Erstellung der Artikel wird nicht nur auf vorhandene Literatur zurückgegriffen. Quellensuche und -auswertung auch in überregionalen Archiven und Bibliotheken ist grundlegend; neben dem Verlagsarchiv André in Offenbach und den digitalisierten Beständen des Schottverlags in München ${ }^{3}$ und Berlin ${ }^{4}$ sind es Stadt- und Staatsarchive in den genannten Städten, die vor allem für die Recherche nach unbekannten Personen hilfreich sind. Die Nutzung von Kirchenbüchern, Zivilstandsregistern, Korrespondenzen, Musikalien, zeitgenössischen Zeitungen/ Zeitschriften, Lexika und diverser Archivbestände ist dabei unerlässlich und gewinnbringend. Insbesondere die Portraitsammlung Manskopf ${ }^{5}$ in der UB Frankfurt a. M. bietet reiches Material für die Bebilderung der Artikel, aber auch selbst fotografierte (Grab-) Denkmäler sowie Titelseiten zeitgenössischer Notendrucke dienen der Veranschaulichung.

\section{Technische Umsetzung und „Netzwerke“}

Die Open-Source-Software DokuWiki ${ }^{6}$ bildet die technische Grundlage, mit der sich unser Online-Lexikon mit überschaubarem Aufwand und ohne tiefgreifende Informatikkenntnisse einrichten ließ. Mit Hilfe interner Verlinkungen wird der hohe Grad der Vernetzung zwischen Einzelpersonen und Institutionen (auch über Stadt- und Landesgrenzen hinaus) sichtbar: Sei es nun als Widmungsträgerin einer Komposition, als Ehemann der klavierspielenden Schwester, Orchester- und andere Arbeitskollegen sowie Amtsnachfolger in verschiedensten Positionen.

3 Bayerische Staatsbibliothek, Erschließung, Digitalisierung und Online-Präsentation des Historischen Archivs des Musikverlags Schott, <https://www.bsb-muenchen.de/ueber-uns/projekte/erschliessung-digitalisierung-undonline-praesentation-des-historischen-archivs-des-musikverlags-schott/> (20.05.2020).

4 Staatbibliothek zu Berlin - Preußischer Kulturbesitz, Erschließung, Digitalisierung und Online-Präsentation des Historischen Archivs des Musikverlags Schott, <https://staatsbibliothek-berlin.de/die-staatsbibliothek/abteilungen/musik/projekte/dfg-projekt-historisches-archiv-des-musikverlags-schott/> (20.05.2020).

5 Universitätsbibliothek J. C. Senckenberg, Porträtsammlung Friedrich Nicolas Manskopf, <https://www.ub.unifrankfurt.de/musik/manskopf_portraets.html> (20.05.2020).

6 DokuWiki, <https://www.dokuwiki.org/dokuwiki> (20.05.2020). 
Dies lässt wiederum Schlüsse zur Lebenswelt und sozialen Einbettung der dokumentierten Personen zu. Externe Links führen zu inhaltlich verwandten Onlinelexika, ${ }^{7}$ digitalisiertem Archivmaterial ${ }^{8}$ und in einzelne Datensätze des RISM-Opac. Insbesondere Informationen zu Drucken (RISM-Serie A/I) werden entsprechend der neuen Kenntnisse zugleich überarbeitet. ${ }^{9}$

\section{Ausblick}

Auch in Zukunft werden wir uns um Zusammenarbeit mit weiteren Forschungsinstituten und Projekten bemühen. Bereits stark fortgeschritten ist die Nutzung unserer Artikel für die Überarbeitung in Personen- und Körperschaftsnormdatensätzen bei RISM (aktuell zitiert bei 225

\section{Einzig, Leopold}

Name: Einzig, Leopold(1856-1906)

Gender: Male

Place: Liblice, Frankfurt am Main

Profession: Arranger, Music teacher, Composer, Violinist

Years active: 27.09.1856-16.01.1906

Lexica: DEUMM/b: 0

EitnerQ: 0

Frank-AltmannTL ${ }^{15}: 0$

Grove $^{5}: 0$

Grove $^{6}: 0$

LedeburTLB: 0

MCL: 0

MGG suppl.: 0

MGG: 0

MMM2 online: Einzig, Leopold [27.09.1856-16.01.1906]

RISM A/l suppl.: 0

RISM A/l: 0

RiemannL ${ }^{12} / p$ suppl.: 0

RiemannL ${ }^{12} / \mathrm{p}: 0$

Other Name: Einzig, Leo

Links: Krämer, Kristina: Art. EINZIG, LEOPOLD, in: MMM2 online, Zugriff am 30.12.2019 GND: https://d-nb.info/gnd/116425954

Get titles containing this id

Abbildung 2: Personendatensatz zu Leopold Einzig im RISM-Opac
Personen und 10 Verlagen). Zahlreiche

Personen sind nur in wenigen, zumeist älteren Lexika oder an keiner vergleichbaren Stelle zu finden (vgl. Abbildung 2 bezüglich des Personendatensatzes zu Leopold Einzig im RISM-Opac). Dies trifft insbesondere, aber nicht ausschließlich, für Personen des 19. und frühen 20. Jahrhunderts zu. Um bei besagtem Beispiel zu bleiben: Während es Literatur zu Leopold Einzigs Sohn Albert und auch zur Tochter Mathilde gibt, wurde dem Vater bisher keine Aufmerksamkeit geschenkt.

Aktuell bemühen wir uns, unsere Quellenangaben zu vorhandenen Korrespondenzen um Verlinkungen zu Kalliope zu ergänzen. Dadurch sollen unse-

7 Wie beispielsweise die frei zugänglichen Onlinelexika: Frankfurter Personenlexikon <https://frankfurterpersonenlexikon.de/>, Stadtlexikon Darmstadt <https://www.darmstadt-stadtlexikon.de/>, Bayerisches MusikerLexikon Online <http://bmlo.de>, Lexikon verfolgter Musiker und Musikerinnen der NS-Zeit <https://www.lexm. uni-hamburg.de/>, Musikvermittlung und Genderforschung: Musikerinnen-Lexikon und multimediale Präsentationen <https://mugi.hfmt-hamburg.de> (sämtlich: 20.05.2020).

8 Hier ist besonders das Hessische Staatsarchiv Darmstadt (HStAD) mit den zum Großherzoglichen Hausarchiv gehörenden Akten der Hofhaltung und des Hofmarschallamts (D 8) sowie der Kabinettsregistratur (D 12) zu nennen.

9 Dass davon mitunter auch Handschriften betroffen sind, zeigen folgende Beispiele: Kristina Krämer, „In Frankfurt a/m zu haben bey Musikus Ludwig': Zur Identifizierung des Kopisten Johann Wilhelm Ludwig", in: RISM News, <http://www.rism.info/de/startseite/newsdetails/browse/1/article/64/in-frankfurt-am-zu-haben-bey-musikusludwig-identifying-the-copyist-johann-wilhelm-ludwig.html> (09.03.2020) und Martin Bierwisch, Kristina Krämer, "Opernpartiturabschriften in der Library of Congress und ein identifizierter Kopist", in: ebd., <http://www. rism.info/de/startseite/newsdetails/browse/1/article/64/copies-of-opera-scores-at-the-library-of-congressand-an-identified-copyist.html> (24.02.2020). 
re Leser*innen Zugriff auf den aktuellen Erfassungsstand erhalten. Im Artikel zu Robert Emil Bockmühl ist dies schon zu sehen, wobei hier auch Briefe aufgeführt sind, die nicht in Kalliope erfasst wurden. ${ }^{10}$ Des Weiteren gibt es Pläne zur Verbesserung der gemeinsamen Normdatei (GND) für Personen und Körperschaften zum Nutzen im Katalogisierungs- und Forschungsalltag von Bibliothekar*innen und Musikwissenschaftler*innen. Daneben wird die Anbindung an die Lehre mit Schwerpunkt Quellenkunde in Form einer weiteren Übung (WiSe 2020/21) fortgesetzt.

Die Zahl der noch zu schreibenden Artikel ist beträchtlich und allein deshalb nicht guten Gewissens benennbar, da das Vordringen in bisher unberücksichtigte Regionen, Institutionen und biographische Bereiche immer wieder gleichsam Lawinen auslöst, die aber keineswegs als bedrohlich empfunden werden, sondern die Entdeckerfreude im Blick auf verschüttete Fakten und Zusammenhänge, vollkommen vergessene Personen und Kompositionen anstacheln. Unterstützung und tatkräftiges Interesse "von außen" sind immer willkommen, und sei es in Form von Hinweisen auf Fehler, Versäumnisse und dergleichen mehr.

Zitation: Axel Beer, Martin Bierwisch, Kristina Krämer, „Das MMM2 - Ein regionalgeschichtliches Onlinelexikon der Arbeitsgemeinschaft für mittelrheinische Musikgeschichte", in: Brückenschläge zwischen Musikwissenschaft und Informatik. Theoretische und praktische Aspekte der Kooperation, in Verbindung mit der Fachgruppe Digitale Musikwissenschaft hrsg. von Stefanie Acquavella-Rauch, Andreas Münzmay und Joachim Veit (= Musikwissenschaft: Aktuelle Perspektiven. Bericht über die Jahrestagung der Gesellschaft für Musikforschung 2019 in Paderborn und Detmold, Bd. 3), Detmold, Musikwissenschaftliches Seminar der Universität Paderborn und der Hochschule für Musik Detmold, 2020, S. 199-205, DOI: 10.25366/2020.108

10 Axel Beer, Alfred Richter, Sebastian Schertel, Art. „Bockmühl, Robert”, in: MMM2, <http://mmm2.mugemir.de/ doku.php?id=bockmuehl $>$ (20.05.2020). 


\section{Abstract}

Based on two printed volumes Musik und Musiker am Mittelrhein (1974, 1981), the online encyclopaedia MMM2 (published in 2018) continues the original idea of a biographical and bibliographical documentation of musicians and musical sources in the middle rhine region. Exploring local music history often means to venture off the beaten track of well-known names and institutions. While perhaps not considered a worthwhile undertaking by some, the 570 articles on musicians, composers, publishers etc. that can currently be found in MMM2 are proof of networks that have hitherto been unknown or disregarded. Filling the gaps in our knowledge of musical life and culture in this area also enables us to improve data such as catalogue entries and name authority files in the RISM database.

\section{Kurzviten}

Axel Beer, geboren 1956 in Fulda. Studium in Frankfurt a. M. (Musikwissenschaft, Lateinische Philologie, Historische Hilfswissenschaften). Magisterexamen 1985, Promotion 1987; 19871995 Wissenschaftlicher Mitarbeiter an der Westfälischen Wilhelms-Universität Münster. Habilitation 1995; seitdem Professor für Musikwissenschaft an der Johannes Gutenberg-Universität Mainz.

Martin Bierwisch studierte Musikwissenschaft und Philosophie in Mainz. Masterexamen 2018 zum Darmstädter Musikverleger Alisky. Seit WiSe 2018/19 wissenschaftlicher Mitarbeiter und Promotionsstudium. Daneben seit 2017 Mitarbeiter in der RISM-Zentralredaktion Frankfurt a. M. für die Überarbeitung von B/I (Sammeldrucke des 16./17. Jahrhunderts). Außerdem Beiratsmitglied und Webmaster der Arbeitsgemeinschaft für mittelrheinische Musikgeschichte e.V. $<$ www.mugemir.de $>$.

Kristina Krämer studierte Musikwissenschaft und British Studies in Mainz. 2019 Abschluss des Masterstudiums mit einer Arbeit zum Klaviervirtuosen Alexander Dreyschock. Seit 2017 wissenschaftliche Hilfskraft für Prof. Dr. Axel Beer, seit 2019 Mitarbeiterin der RISM-Zentralredaktion und Promotionsvorhaben zum Musikverlag Schott im ersten Viertel des 19. Jahrhunderts. 


\section{Das MMM2 - Ein regionalgeschichtliches Onlinelexikon der Arbeitsgemeinschaft für mittelrheinische Musikgeschichte}

\begin{abstract}
Wen findet man im Lexikon?
Komponisten*innen, Instrumentenbauer*innen, Musikpädagogen*innen, Wissenschaftler*innen oder auch Verleger*innen, die im mittelrheinischen Raum nicht nur tätig waren, sondern auch „Werke“ in einem umfänglichen Sinne hinterlassen haben. Hierbei ist neben der musikalischen „Prominenz“ der Region ein vollkommen vergessener und in keinem anderen Lexikon zu findender Mainzer Musiklehrer, wie etwa Frohwald Thiemer, von dem eine Handvoll Kompositionen zu ermitteln war, ebenso selbstverständlich enthalten wie ein Niccolò Paganini, der in der Region konzertierte und einige Monate in Frankfurt weilte - dass Personen von überregionaler und allgemeiner Bekanntheit wie etwa Letzterer nur hinsichtlich ihrer Tätigkeit oder ihrer Präsenz im mittelrheinischen Raum Berücksichtigung finden, sei betont. Lebende Personen werden nicht berücksichtigt.
\end{abstract}

\section{Geographische Abgrenzung}

Der Raum umfasst zunächst das gesamte heutige Bundesland Rheinland-Pfalz, ferner das Saarland, von Hessen den Rheingau und Teile des ehemaligen Fürstentums Nassau, das frühere Großherzogtum Hessen-Darmstadt, von Baden-Württemberg die ehemaligen Teile der Kurpfalz, schließlich die im ehemaligen „Oberstift“ des Kurfürstentums Mainz in Bayern (Unterfranken) gelegenen Gebiete. Städtische Zentren sind also folglich: Mainz, Wiesbaden, Frankfurt a/M., Offenbach, Darmstadt, Aschaffenburg, Worms, Speyer, Koblenz und Trier.

\section{Netzwerke}

Mit Hilfe interner Verlinkungen wird der hohe Grad der Vernetzung zwischen Einzelpersonen und Institutionen (auch über Stadt- und Landesgrenzen hinaus) sichtbar: Sei es nun als Widmungsträgerin einer Komposition, als Ehemann der klavierspielenden Schwester, Orchester- und andere Arbeitskollegen sowie Amtsnachfolger in verschiedensten Positionen. Dies lässt wiederum Schlüsse zur Lebenswelt und sozialen Einbettung der betreffenden Person zu. Nebenstehende Graphik zeigt die Verlinkungen aller Artikel, die sich, ausgehend von Joachim Raff, in maximal drei Schritten erreichen lassen.

\section{Quellen}

Für die Erstellung der Artikel wird nicht nur auf vorhandene Literatur zurückgegriffen. Quellensuche und -auswertung auch in überregionalen Archiven und Bibliotheken ist grundlegend; neben dem Verlagsarchiv André in Offenbach und den digitalisierten Beständen des Schottverlags in München und Berlin sind es Stadt- und Staatsarchive in den genannten Städten, welche vor allem für die Recherche nach unbekannten Personen hilfreich sind. Die Nutzung von Kirchenbüchern, Zivilstandsregistern, Korrespondenzen, Musikalien, zeitgenössischen Zeitungen/Zeitschriften, Lexika und diverser Akten ist dabei unerlässlich und gewinnbringend. Insbesondere die Portraitsammlung Manskopf in der UB Frankfurt/M. bietet reiches Material für die Bebilderung der Artikel, aber auch selbst photographierte Grab- und Denkmäler der Region sowie zeitgenössische Titelillustrationen der Kompositionen dienen der Veranschaulichung.

\section{Technische Umsetzung}

Die Open-Source-Software „DokuWiki“ bildet die technische Grundlage, mit der sich unser Online-Lexikon mit überschaubarem Aufwand und ohne tiefgreifende Informatikkenntnisse einrichten ließ. Auch für das Einstellen neuer Artikel ist nur eine kurze Einarbeitung nötig.

\section{Ausblick}

Auch in Zukunft werden wir uns um Zusammenarbeit mit weiteren Forschungsinstituten und Projekten bemühen. Neben der bereits begonnenen Einarbeitung von Daten in Personennormdatensätzen bei RISM gibt es Pläne zur Verbesserung der gemeinsamen Normdatei (GND) für Personen und Körperschaften. Ziel und Zweck der Kooperationen sind Nachnutzung und Nutzerfreundlichkeit für Bibliothekar*innen und Musikwissenschaftler*innen. Daneben wird die Anbindung an die Lehre mit Schwerpunkt Quellenkunde in Form einer weiteren Master-Übung (WiSe 2019/20) fortgesetzt.

\section{$M_{\text {Iir }}^{\text {war }}$ usiker ittelrhein}

\section{Daten und Fakten}

1974, 1981: Vorgänger (MMM1) - Zwei von Hubert Unverricht herausgegebene Lexikonbände mit insgesamt 83 Artikeln

2001-2008 Vorarbeiten zu einer gedruckten Fortsetzung

2017 Entscheidung zur Onlinepublikation

Winter 2017/18 Erstellung der Website

2018 Revision alter sowie Erstellung neuer Artikel

Online seit Oktober 2018 mit 250 Artikeln

WiSe 2018/19 Master-Übung „Ein Lexikon entsteht“ (JGU Mainz)

Juli 2019: 400 Artikel online

Aktueller Stand (Sept. 2019): 450 Artikel

Herausgeber: Prof. Dr. Axel Beer (JGU Mainz)

Mitarbeiter: Martin Bierwisch M. A., Kristina Krämer M. A. Das Lexikon entsteht im Auftrag der Arbeitsgemeinschaft für mittelrheinische Musikgeschichte unter Mitarbeit zahlreicher Musik- und Lokalgeschichtsforscher*innen.
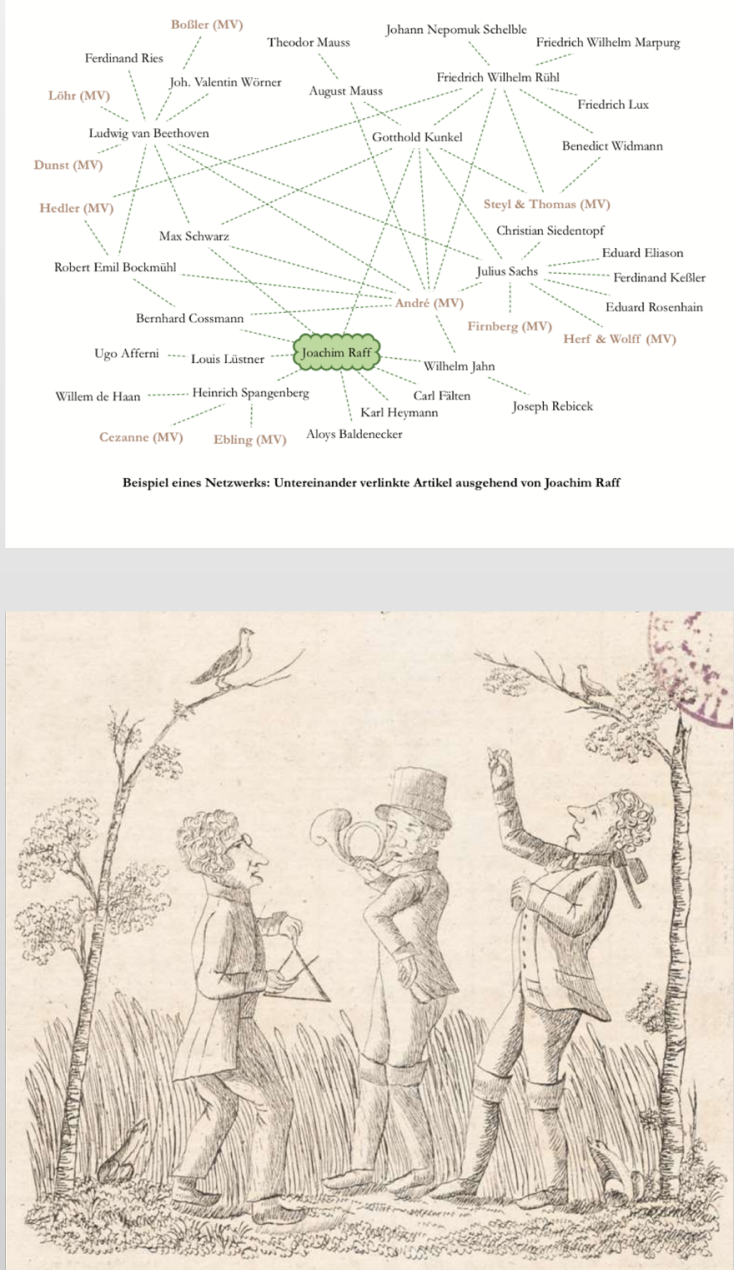


\section{Brückenschläge zwischen Musikwissenschaft und Informatik}

Theoretische und praktische Aspekte der Kooperation

Herausgegeben von Stefanie Acquavella-Rauch,

Andreas Münzmay und Joachim Veit 
Brückenschläge zwischen Musikwissenschaft und Informatik 


\section{Musikwissenschaft: Aktuelle Perspektiven}

Bericht über die Jahrestagung der Gesellschaft für Musikforschung 2019 in Paderborn und Detmold

Herausgegeben von Rebecca Grotjahn und Nina Jaeschke

Band 3 


\section{Brückenschläge zwischen Musikwissenschaft und Informatik}

Theoretische und praktische Aspekte der Kooperation

Beiträge der Symposien zur Digitalen Musikwissenschaft

Osnabrück 2018 und Paderborn 2019

im Rahmen der Jahrestagungen der Gesellschaft für Musikforschung

In Verbindung mit der Fachgruppe Digitale Musikwissenschaft herausgegeben von

Stefanie Acquavella-Rauch, Andreas Münzmay und Joachim Veit

Detmold: Musikwissenschaftliches Seminar der Universität Paderborn und der Hochschule für Musik Detmold 2020 
DOI: $10.25366 / 2020.87$

Online-Version verfügbar unter der Lizenz: Urheberrecht 1.0, $<$ https://rightsstatements.org/page/InC/1.0/?language =de >

Bibliografische Information der Deutschen Nationalbibliothek

Die Deutsche Nationalbibliothek verzeichnet diese Publikation in der Deutschen Nationalbibliografie; detaillierte bibliografische Daten sind im Internet über http://dnb.d-nb.de abrufbar.

\section{Impressum}

Redaktion: Stefanie Acquavella-Rauch, Andreas Münzmay und Joachim Veit Satz: Nina Jaeschke und Joachim Veit

(C) Musikwissenschaftliches Seminar der Universität Paderborn und der Hochschule für Musik Detmold 2020 


\section{INHALT}

Rebecca Grotjahn, Nina Jaeschke

Vorwort zu Band 1-3

IX

Stefanie Acquavella-Rauch, Andreas Münzmay, Joachim Veit

Brückenschläge zwischen Musikwissenschaft und Informatik - Vorbemerkung

$\mathrm{XI}$

\section{KOLLABORATIONEN - KO-LABORATORIEN}

\section{Reinhard Keil}

Der Computer als Denkzeug für hermeneutische Arbeit

\section{Ulrich Konrad}

Philologie und Digitalität. Perspektiven für die Musikwissenschaft im Kontext fächerübergreifender Institutionen

\section{Gudrun Oevel}

Infrastruktureinrichtungen in Forschungsprojekten - Spagat oder Chance?

\section{Dennis Ried}

Erhebung, Transformation und Präsentation digitaler Forschungsdaten

Anna Neovesky, Frederic von Vlahovits

IncipitSearch - Leitfaden zur Zusammenarbeit

Elisabeth Treydte

Clara Schumann \#digital. 40 Jahre Archiv Frau und Musik und der Start in die Digitalisierung

\section{TEXT/DATEN/PROZESSE}

\section{Christine Siegert}

Komponisten-Gesamtausgaben im digitalen Zeitalter: Perspektiven

und Reflexionen am Beispiel Ludwig van Beethovens

Markus Neuwirth, Johannes Hentschel, Martin Rohrmeier

Perspectives of Musical Corpus Studies: The Annotated Mozart Sonatas

Agnes Amminger, Franz Kelnreiter

Leopold Mozarts „Gründliche Violinschule". Zur Textcodierung und -präsentation einer digitalen Edition

\section{Oleksii Sapov}

Algorithmische Automatisierung komplexer Notationsregeln in MEI-XML am Beispiel von Versetzungszeichen 


\section{Susanne Cox, Richard Sänger}

Digitale Fassungsvergleiche am Beispiel von Beethovens Eigenbearbeitungen

Agnes Seipelt

Digitale Edition und Harmonische Analyse mit MEI von Anton Bruckners

Studienbuch

Stefanie Acquavella-Rauch

Musikalische Schaffensprozesse 2.0 - Inkorporation audiovisueller Medien

der populären Musik in Methoden der digitalen Edition

\section{DIGITAL(ISIERT)E MATERIALITÄTEN}

\section{Miriam Akkermann}

(Musik)Instrument (im) Computer

\section{Daniel Fütterer}

Herausforderungen bei der Kodierung von Paratext am Beispiel Neuer Musik mit Live-Elektronik

\section{Matthias Pasdzierny}

How much is the glitch? Das digitale Paradigma als Herausforderung

und Chance für die historische Musikwissenschaft

\section{Shintaro Miyazaki}

Musik für Maschinen?! - Wo sich die Wissenschaft der Medien, des Computers und der Musik treffen und wie sie zusammenarbeiten könnten

\section{MUSIKGESCHICHTE(N) IM NETZ}

\section{Matthias Tischer}

Musikgeschichte der DDR: Ein Pilotprojekt zur digitalen Musikvermittlung

Annette van Dyck-Hemming, Jan Eberhardt, Melanie Wald-Fuhrmann

Ansätze zur Analyse historischer Netzwerke mit Neo4j® - Aus der Projekt-Werkstatt der Datenbank zur Fachgeschichte der Musikwissenschaft

Axel Beer, Martin Bierwisch, Kristina Krämer

Das MMM2 - Ein regionalgeschichtliches Onlinelexikon der Arbeitsgemeinschaft für mittelrheinische Musikgeschichte

\section{Matej Santi}

Was erzählt Fritz Kreislers Geige?

\section{Elias Berner}

Alle Menschen werden Brüder?! Ein historisches Dokument aus dem

Nationalsozialismus in den sozialen Medien 
Gabriele Buschmeier in memoriam 